\title{
DECOMPOSITION OF NONNEGATIVE GROUP-MONOTONE MATRICES
}

\author{
BY
}

S. K. JAIN, ${ }^{1}$ EDWARD K. KWAK AND V. K. GOEL

\begin{abstract}
A decomposition of nonnegative matrices with nonnegative group inverses has been obtained. This decomposition provides a new approach to the solution of problems relating to nonnegative matrices with nonnegative group inverses. As a consequence, a number of results are derived. Our results, among other things, answer a question of Berman, extend the theorems of Berman and Plemmons, DeMarr and Flor.
\end{abstract}

1. Introduction. Let $A$ be an $m \times n$ real matrix. Consider the equations: (1) $A X A=A$, (2) $X A X=X$, (3) $(A X)^{T}=A X$, (4) $(X A)^{T}=X A$, and (5) $A X=X A$ where $X$ is an $n \times m$ real matrix and $T$ denotes the transpose. For a rectangular matrix $A$ and for a nonempty subset $\lambda$ of $\{1,2,3,4,5\}, X$ is called a $\lambda$-inverse of $A$ if $X$ satisfies equations ( $i$ ) for each $i \in \lambda$. In particular, the $\{1,2,3,4\}$-inverse of $A$ is the unique Moore-Penrose generalized inverse and is denoted by $A^{\dagger}$. $A\{1,2\}$-inverse of $A$ which satisfies (5) is necessarily square and is called a group inverse. The group inverse of a matrix $A$, if it exists, is unique and is denoted by $A^{\#}$.

A matrix $A$ is called group-monotone if $A^{\#}$ exists and is nonnegative. A matrix $A=\left(a_{i j}\right)$ is called 0 -symmetric if $a_{i j}=0$ implies $a_{j i}=0$. Thus every symmetric matrix and every positive matrix is 0 -symmetric. $A$ is called range-Hermitian (also called EPr) if the range of $A$ is equal to the range of $A^{T}$, i.e., $R(A)=R\left(A^{T}\right) . A$ is range-Hermitian if and only if $A A^{\dagger}=A^{\dagger} A$ and so $A^{\dagger}=A^{\sharp}$. An $m \times n$ matrix $A=\left(a_{i j}\right)$ is called row (or column) stochastic if $a_{i j}>0$ and $\sum_{j=1}^{n} a_{i j}=1,1<i<n$ (or $\sum_{i=1}^{n} a_{i j}=1,1<j<n$ ). If a matrix $A$ is a direct sum of matrices $S_{i}$, then $S_{i}$ will be called summands of $A$. A nonzero matrix $A$ is called a zero divisor if $A B=0$ or $B A=0$ for some nonzero matrix $B$. For all other terminology the reader is referred to Ben-Israel and Greville [1].

Theorem 1 of this paper characterizes all nonnegative matrices $A$ which have nonnegative group inverses; equivalently, $A^{(1,2)}=p(A)>0$, where $p(A)$ is a polynomial in $A$ with scalar coefficients. This theorem generalizes the known results for nonnegative matrices $A$ whose $A^{\dagger}$ is $A$ [2] or, more generally, $A^{\dagger}$ is some polynomial in $A$ [7]. The solution to the problem raised by Berman of the characterization of all nonnegative matrices which are equal to a $\{1\}$ - or $\{1,2\}$-inverse of themselves also comes as a special case of Theorem 1. As a consequence of Theorem 1, we show (Corollary 2) that if $A$ is a nonnegative matrix with $A^{m}=A$, $m>2$, then $A=A_{1}+A_{2}+\cdots+A_{k}$, where $A_{i}>0 ; A_{i}^{m}=A_{i} ; A_{i} A_{j}=0, i \neq j$;

Received by the editors May 15, 1978.

AMS (MOS) subject classifications (1970). Primary 15A09, 15A48; Secondary 15A51.

${ }^{1}$ Supported by OUR grant No. 551. 
$d_{i}=\operatorname{rank} A_{i}, d_{i} \mid m-1$. This generalizes the theorem of DeMarr [5] for nonnegative idempotent matrices. Corollary 3 (Corollary 4) of Theorem 2 shows that for a nonnegative range-Hermitian (row stochastic) matrix $A$ with $A^{\sharp}>0, A^{\sharp}=A^{\dagger}=$ $H A^{m}=A^{m} H\left(A^{\#}=A^{\dagger}=A^{m}\right)$ where $H$ is a diagonal matrix with all entries positive. Theorem 4 characterizes all nonnegative rank factorizations of nonnegative group-monotone matrices. Theorems of Berman and Plemmons [3, Theorem 2 and Theorem 3] are also consequences of the characterizations obtained in Theorem 4. Our results, among others, depend on the following theorems proved in [6] and [7].

TheOReM A ([6, TheOREM 2]). If $E$ is a nonnegative idempotent matrix of rank $r$ with no row or column completely zero. Then there exists a permutation matrix $P$ such that

$$
P E P^{T}=\left(\begin{array}{ccc}
x_{1} y_{1}^{T} & & 0 \\
& \ddots & \\
0 & & x_{r} y_{r}^{T}
\end{array}\right)
$$

where $x_{i}, y_{i}$ are positive vectors with $y_{i}{ }^{T} x_{i}=1$. In particular, $E$ is 0 -symmetric.

TheOREM B ([7, REMARK 3]). Let $A$ be a nonnegative matrix and $p(A)=\alpha_{1} A^{m_{1}}$ $+\cdots+\alpha_{k} A^{m_{k}}, \alpha_{i} \neq 0, m_{i}>0$, such that $p(A)>0, A p(A)$ is 0-symmetric, $A p(A) A$ $=A$, and $\operatorname{rank} A=\operatorname{rank} p(A)$. Then there exists a permutation matrix $P$ such that $P A P^{T}$ is a direct sum of matrices of the following three types (not necessarily all)

(I) $\beta x y^{T}$, where $x$ and $y$ are positive vectors with $y^{T} x=1$, and $\beta$ is some positive number satisfying $\sum_{m_{i}} \alpha_{i} \beta^{m_{i}+1}=1$ :

(II)

$$
\left(\begin{array}{ccccc}
0 & \beta_{12} x_{1} y_{2}^{T} & 0 & \ldots & 0 \\
0 & 0 & \beta_{23} x_{2} y_{3}^{T} & \ldots & \ldots \\
0 & 0 & 0 & \ldots & \beta_{d-1 d} x_{d-1} y_{d}^{T} \\
\beta_{d 1} x_{d} y_{1}^{T} & 0 & 0 & \ldots & 0
\end{array}\right)
$$

where $x_{i}$ and $y_{i}$ are positive vectors of the same order with $y_{i}{ }^{T} x_{i}=1, x_{i}$ and $x_{j}, i \neq j$, are not necessarily of the same order, and $\beta_{12}, \beta_{23}, \ldots, \beta_{d 1}$ are arbitrary positive numbers with $d>1$ and $d \mid m_{i}+1$ for some $m_{i}$ such that the product $\beta_{12} \beta_{23} \ldots \beta_{d 1}$ is a common root of the following system of at most $d$ equations in $t$ :

$$
\begin{gathered}
\sum_{d \mid\left(m_{i}+1\right)} \alpha_{i} t^{\left(m_{i}+1\right) / d}=1, \\
\sum_{d \mid\left(m_{i}+1-k\right)} \alpha_{i} t^{\left(m_{i}+1-k\right) / d}=0, \quad k \in\{1, \ldots, d-1\},
\end{gathered}
$$


where the summation in each of the above equations runs over all those $m_{i}$ for which $d \mid\left(m_{i}+1-k\right), k=0,1, \ldots, d-1$, with the convention that if there is no $m_{i}$ for which $d \mid\left(m_{i}+1-k\right), k \in\{1, \ldots, d-1\}$, then the corresponding equation is absent.

(III) A zero matrix.

In particular, if all $\alpha_{i}>0$ then $\beta$ in type (I) and the product $\beta_{12} \beta_{23} \ldots \beta_{d 1}$ in type (II) are unique. Further, in this case the positive integer $d$, i.e. the rank of a matrix of type (II), must divide each $m_{i}+1$.

The concept of 0-symmetry has played a crucial role in the development of this paper.

2. Main results. Let $A$ be any $n \times n$ matrix. Let us group the indices $i=$ $1,2,3, \ldots, n$ into four disjoint sets according to whether the $i$ th row and the $i$ th column of $A$ are both zero, or the $i$ th row is zero but the $i$ th column is not, and so on. Then by simultaneously rearranging rows and columns, we can find a permutation matrix $P$ such that

$$
P A P^{T}=\left(\begin{array}{cccc}
K & L & 0 & 0 \\
0 & 0 & 0 & 0 \\
M & N & 0 & 0 \\
0 & 0 & 0 & 0
\end{array}\right)
$$

where the diagonal blocks are square matrices such that $K$ and $L$ have no zero rows in common, and $K$ and $M$ have no zero columns in common. It may be noted that in certain situations some of the blocks may be absent. For example, if $A$ is row stochastic then

$$
P A P^{T}=\left(\begin{array}{cc}
K & 0 \\
M & 0
\end{array}\right)
$$

In view of the frequent use of the above representation of a matrix throughout this paper, we record it in the following lemma.

LEMma 1. Let $A$ be a square matrix. Then there exists a permutation matrix $P$ such that

$$
P A P^{T}=\left(\begin{array}{cccc}
K & L & 0 & 0 \\
0 & 0 & 0 & 0 \\
M & N & 0 & 0 \\
0 & 0 & 0 & 0
\end{array}\right)
$$

where $K$ and $L$ have no zero rows in common, and $K$ and $M$ have no zero columns in common. 
THEOREM 1. Let $A$ be a nonnegative matrix and $A^{(1,2)}=p(A)>0$, where $p(A)=$ $\alpha_{1} A^{m_{1}}+\cdots+\alpha_{k} A^{m_{k}}, \alpha_{i} \neq 0, m_{i}>0$. Then there exists a permutation matrix $P$ such that

$$
P A P^{T}=\left(\begin{array}{cccc}
J & J D & 0 & 0 \\
0 & 0 & 0 & 0 \\
C J & C J D & 0 & 0 \\
0 & 0 & 0 & 0
\end{array}\right),
$$

where $C, D$ are some nonnegative matrices of appropriate sizes and $J$ is a direct sum of matrices of the following types (not necessarily both):

(I) $\beta x y^{T}$, where $x$ and $y$ are positive vectors with $y^{T} x=1$ and $\beta$ is a positive root of

$$
\sum_{m_{i}} \alpha_{i} t^{m_{i}+1}=1
$$

(II)

$$
\left[\begin{array}{cccccc}
0 & \beta_{12} x_{1} y_{2}^{T} & 0 & 0 & \ldots & 0 \\
0 & 0 & \beta_{23} x_{2} y_{3}^{T} & 0 & \ldots & 0 \\
\ldots & \ldots & \ldots & \ldots & \ldots & \ldots
\end{array}\right]
$$

where $x_{i}$ and $y_{i}$ are positive vectors of the same order with $y_{i}{ }^{T} x_{i}=1 ; x_{i}$ and $x_{j}, i \neq j$, are not necessarily of the same order; and $\beta_{12}, \ldots, \beta_{d 1}$ are arbitrary positive numbers with $d>1$ and $d \mid m_{i}+1$ for some $m_{i}$ such that the product $\beta_{12} \beta_{23} \cdots \beta_{d 1}$ is a common root of the following system of at most $d$ equations in $t$

$$
\begin{gathered}
\sum_{d \mid\left(m_{i}+1\right)} \alpha_{i} t^{\left(m_{i}+1\right) / d}=1, \\
\sum_{d \mid\left(m_{i}+1-k\right)} \alpha_{i} t^{\left(m_{i}+1-k\right) / d}=0, \quad k \in\{1,2, \ldots, d-1\},
\end{gathered}
$$

where the summation in each of the above equations runs over all those $m_{i}$ for which $d \mid\left(m_{i}+1-k\right), k=0,1,2, \ldots, d-1$, with the convention that if there is no $m_{i}$ for which $d \mid\left(m_{i}+1-k\right), k \in\{1, \ldots, d-1\}$, then the corresponding equation is absent.

In particular, if all $\alpha_{i}>0$ then $\beta$ in type (I) and the product $\beta_{12} \beta_{23} \cdots \beta_{d 1}$ in type (II) are unique. Further, in this case the positive integer $d$, i.e. the rank of a matrix of type (II), must divide each $m_{i}+1$.

Conversely, if for some permutation matrix $P$

$$
P A P^{T}=\left(\begin{array}{cccc}
J & J D & 0 & 0 \\
0 & 0 & 0 & 0 \\
C J & C J D & 0 & 0 \\
0 & 0 & 0 & 0
\end{array}\right),
$$


where $C, D$ are arbitrary nonnegative matrices of appropriate sizes and $J$ is a direct sum of matrices of the following types (not necessarily both):

(I') $\beta x y^{T}, \beta>0, x, y$ are positive vectors with $y^{T} x=1$.

(II')

$$
\left(\begin{array}{cccccc}
0 & \beta_{12} x_{1} y_{2}^{T} & 0 & 0 & \ldots & 0 \\
0 & 0 & \beta_{23} x_{2} y_{3}^{T} & 0 & \ldots & \ldots \\
0 & 0 & 0 & \ldots & 0 & \beta_{d-1 d} x_{d-1} y_{d}^{T} \\
\beta_{d 1} x_{d} y_{1}^{T} & 0 & 0 & \ldots & 0 & 0
\end{array}\right]
$$

where $\beta_{i j}>0, x_{i}$ and $y_{i}$ are positive vectors with $y_{i}{ }^{T} x_{i}=1$, then $A^{(1,2)}>0$ and is equal to some polynomial in $A$ with scalar coefficients.

Proof. By Lemma 1 , there exists a permutation matrix $P_{1}$ such that

$$
P_{1} A P_{1}^{T}=\left(\begin{array}{cccc}
K & L & 0 & 0 \\
0 & 0 & 0 & 0 \\
M & N & 0 & 0 \\
0 & 0 & 0 & 0
\end{array}\right),
$$

where $K, L, M$, and $N$ are nonnegative matrices such that $K$ and $L$ have no zero rows in common, and $K$ and $M$ have no zero columns in common. Since $A^{(1,2)}=p(A)$, we have $A^{2} p(A)=A$ and $A(p(A))^{2}=p(A)$. From $A^{2} p(A)=A$ we obtain $K^{2} p(K)=K, K p(K) L=L, M K p(K)=M$, and $M p(K) L=N$. Hence $K p(K)$ is a nonnegative idempotent matrix. Since $K p(K) K=K$ and $K p(K) L=L$ have no zero rows in common, $K p(K)$ cannot have a zero row. Similarly, no column of $K p(K)$ is zero. Thus by Theorem $A, K p(K)$ is 0-symmetric. Similarly, from $A(p(A))^{2}=p(A)$, we obtain $K(p(K))^{2}=p(K)$, which, together with $K^{2} p(K)$ $=K$, gives $\operatorname{rank} K=\operatorname{rank} p(K)$. But then by Theorem B, there exists a permutation matrix $P_{2}$ such that $P_{2} K P_{2}^{T}$ is a direct sum of matrices of types (I) or (II) (not necessarily both). Set

$$
P=\left(\begin{array}{cc}
P_{2} & 0 \\
0 & I
\end{array}\right) P_{1}
$$

where the block matrices are of suitable orders. Then $P A P^{T}$ is of the desired form.

The converse is trivial.

Corollary 1. Let $A$ be a nonnegative matrix of rank $r$ and let $A^{(1,2)}=p(A)>0$, $p(A)=\alpha_{1} A^{m_{1}}+\cdots+\alpha_{k} A^{m_{k}}, \alpha_{i} \neq 0, m_{i}>0$. Then $A=A_{1}+A_{2}+\cdots+A_{k}$, where $A_{i}>0 ; A_{i} A_{j}=0, i \neq j ; A_{i}^{(1,2)}=p\left(A_{i}\right)$; rank $A_{i}=d_{i}, \sum_{i=1}^{k} d_{i}=r$, and $d_{i}$ divides some $m_{j}+1$.

Proof. By Theorem 1, there exists a permutation matrix $\boldsymbol{P}$ such that

$$
P A P^{T}=\left(\begin{array}{cccc}
J & J D & 0 & 0 \\
0 & 0 & 0 & 0 \\
C J & C J D & 0 & 0 \\
0 & 0 & 0 & 0
\end{array}\right),
$$


where $J$ is a direct sum of the matrices of the types (I) or (II) (not necessarily both) and $C, D$ are nonnegative matrices of appropriate orders. Thus

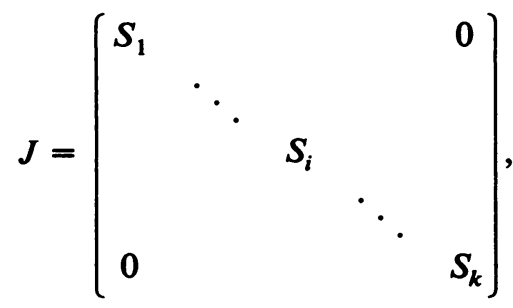

where $S_{i}$ 's are of the types (I) or (II) and rank $S_{i}=d_{i}$. Set

$$
J_{i}=\left(\begin{array}{ccccccc}
0 & & & & & & 0 \\
& \ddots & & & & & \\
& & 0 & & & & \\
& & & S_{i} & & & \\
& & & & 0 & & \\
0 & & & & & \ddots & 0
\end{array}\right) .
$$

Then $A=\sum_{i=1}^{k} A_{i}$, where

$$
A_{i}=P^{T}\left(\begin{array}{cccc}
J_{i} & J_{i} D & 0 & 0 \\
0 & 0 & 0 & 0 \\
C J_{i} & C J_{i} D & 0 & 0 \\
0 & 0 & 0 & 0
\end{array}\right) P
$$

It can be easily verified that $A_{i}>0 ; A_{i} A_{j}=0, i \neq j ; A_{i}^{(1,2)}=p\left(A_{i}\right)$; rank $A_{i}=d_{i}$, $\sum_{i=1}^{k} d_{i}=r$, and $d_{i}$ divides some $m_{j}+1$.

The corollary which follows generalizes DeMarr's theorem for nonnegative idempotent matrices [5].

Corollary 2. Let $A$ be a nonnegative matrix of rank $r$ and let $A=A^{m}$, where $m>2$ is a positive integer. Then $A=A_{1}+A_{2}+\cdots+A_{k}$, where $A_{i}>0 ; A_{i} A_{j}=$ $0, i \neq j ; A_{i}^{m}=A_{i} ; \operatorname{rank} A_{i}=d_{i}, d_{i} \mid m-1, \sum_{i=1}^{k} d_{i}=r$.

Proof. Follows from Corollary 1.

REMARK 1. Theorem 1 provides a complete solution, in a more general case, to the problem raised by Berman of characterization of the class of nonnegative matrices $A$ with $\{1\}$-inverse or $\{1,2\}$-inverse equal to $A$ itself [2, Remark 5].

Henceforth by matrices of types (I) or (II), we will mean the matrices of types (I) or (II) described in Theorem 1.

TheOREM 2. Let $A$ be a nonnegative matrix having a nonnegative group inverse $A^{\sharp}$. Then $A^{\sharp}=K_{1} A^{m}=A^{m} K_{2}$, where

$$
K_{1}=P^{T}\left(\begin{array}{cccc}
K & 0 & 0 & 0 \\
0 & 0 & 0 & 0 \\
C K & 0 & 0 & 0 \\
0 & 0 & 0 & 0
\end{array}\right) P, \quad K_{2}=P^{T}\left(\begin{array}{cccc}
K & K D & 0 & 0 \\
0 & 0 & 0 & 0 \\
0 & 0 & 0 & 0 \\
0 & 0 & 0 & 0
\end{array}\right) P,
$$


$P$ is a permutation matrix, $K$ is a diagonal matrix with positive diagonal entries, $C, D$ are some nonnegative matrices of appropriate sizes, and $m$ is a positive integer. Indeed, we may also choose

$$
K_{1}=K_{2}=P^{T}\left(\begin{array}{cccc}
K & K D & 0 & 0 \\
0 & 0 & 0 & 0 \\
C K & C K D & 0 & 0 \\
0 & 0 & 0 & 0
\end{array}\right) P
$$

Proof. By Theorem 1 there exists a permutation matrix $P$ such that

$$
P A P^{T}=\left(\begin{array}{cccc}
J & J D & 0 & 0 \\
0 & 0 & 0 & 0 \\
C J & C J D & 0 & 0 \\
0 & 0 & 0 & 0
\end{array}\right),
$$

where $C, D$ are some nonnegative matrices of appropriate sizes and $J$ is a direct sum of matrices of types (I) or (II) (not necessarily both). We note that if $S$ is a summand of type (I) then $S^{(1,2)}=\beta^{-1} x y^{T}=\beta^{-2} S=\cdots=\beta^{-k-1} S^{k}$, for any positive integer $k$. We show that if $S$ is a summand of type (II) then $S^{(1,2)}=$ $\left(\beta_{12} \beta_{23} \cdots \beta_{d 1}\right)^{-k} S^{k d-1}$ for any positive integer $k$. A straightforward verification shows that

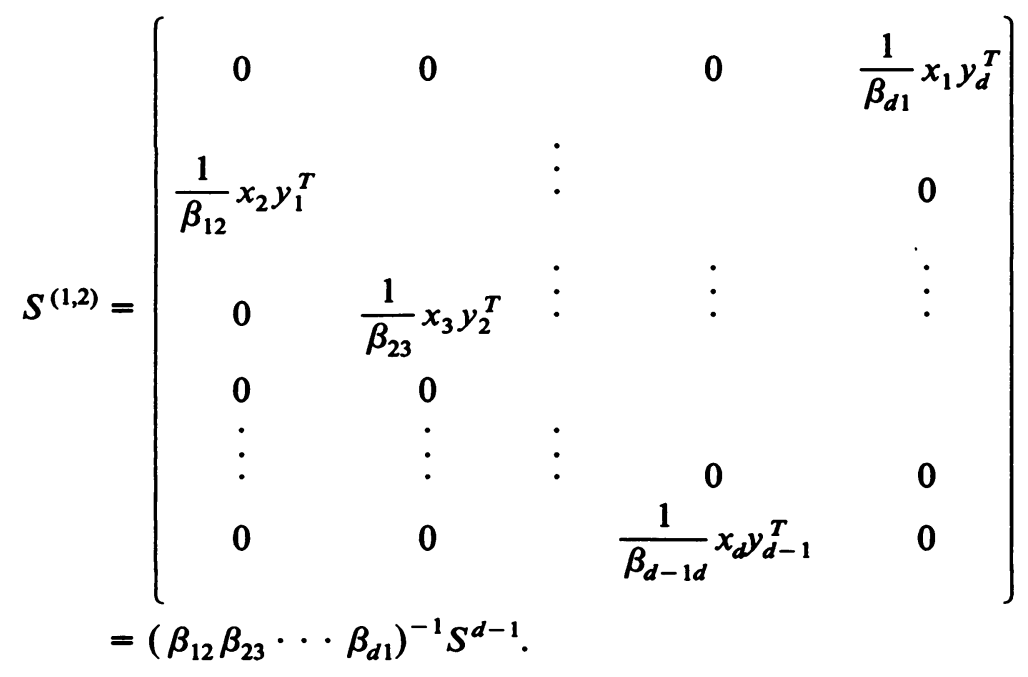

Also

$$
S^{k d}=\left(\beta_{12} \beta_{23} \cdots \beta_{d 1}\right)^{k}\left(\begin{array}{ccc}
x_{1} y_{1}^{T} & & 0 \\
& \ddots & \\
0 & & x_{d} y_{d}^{T}
\end{array}\right)
$$

for any positive integer $k$. Thus $S^{(1,2)}=\left(\beta_{12} \beta_{23} \cdots \beta_{d 1}\right)^{-k} S^{k d-1}$ as asserted. Now if $S_{11}, S_{12}, \ldots, S_{1 r}$ are summands of type (I) and $S_{21}, S_{22}, \ldots, S_{2 s}$ are summands of type (II) of ranks $d_{21}, \ldots, d_{2 s}$ respectively, then 

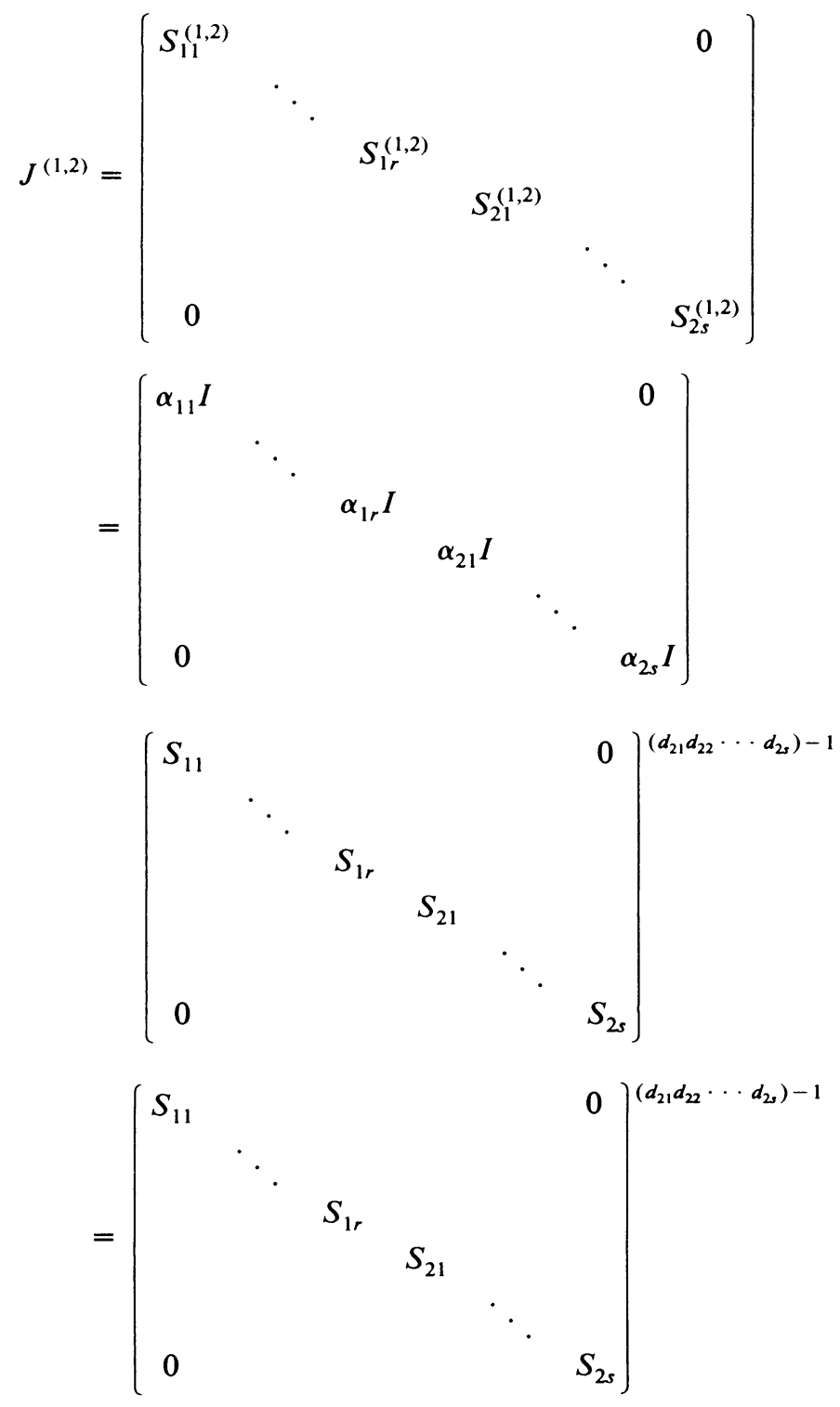

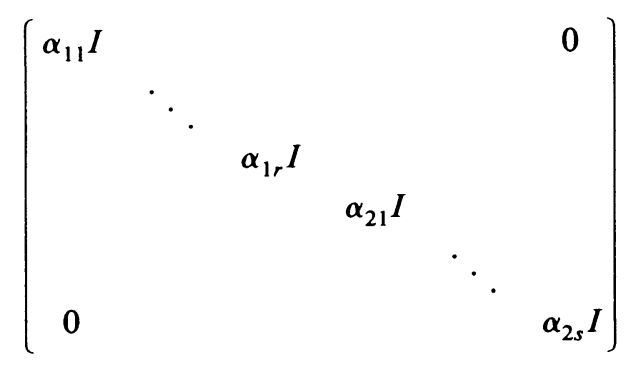

where $\alpha_{i j} I$ are scalar matrices of appropriate sizes, $\alpha_{i j}>0$. Thus $J^{(1,2)}=K J^{m}=$ $J^{m} K$, where 


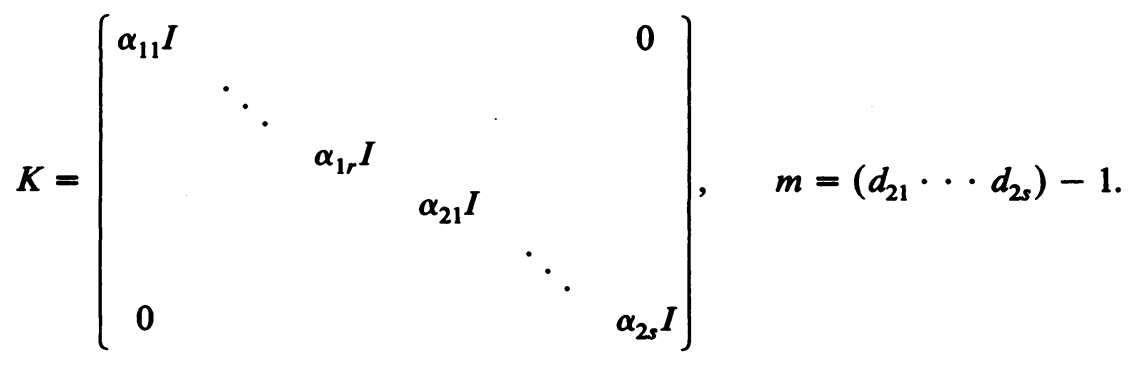

Thus

$$
\begin{aligned}
P A^{(1,2)} P^{T} & =\left[\begin{array}{cccc}
K J^{m} & K J^{m} D & 0 & 0 \\
0 & 0 & 0 & 0 \\
C K J^{m} & C K J^{m} D & 0 & 0 \\
0 & 0 & 0 & 0
\end{array}\right] \\
& =\left(\begin{array}{cccc}
K & 0 & 0 & 0 \\
0 & 0 & 0 & 0 \\
C K & 0 & 0 & 0 \\
0 & 0 & 0 & 0
\end{array}\right]\left[\begin{array}{cccc}
J^{m} & J^{m} D & 0 & 0 \\
0 & 0 & 0 & 0 \\
C J^{m} & C J^{m} D & 0 & 0 \\
0 & 0 & 0 & 0
\end{array}\right] \\
& =\left(\begin{array}{cccc}
J^{m} & J^{m} D & 0 & 0 \\
0 & 0 & 0 & 0 \\
C J^{m} & C J^{m} D & 0 & 0 \\
0 & 0 & 0 & 0
\end{array}\right]\left[\begin{array}{cccc}
K & K D & 0 & 0 \\
0 & 0 & 0 & 0 \\
0 & 0 & 0 & 0 \\
0 & 0 & 0 & 0
\end{array}\right) .
\end{aligned}
$$

Hence

$$
A^{(1,2)}=P^{T}\left(\begin{array}{cccc}
K & 0 & 0 & 0 \\
0 & 0 & 0 & 0 \\
C K & 0 & 0 & 0 \\
0 & 0 & 0 & 0
\end{array}\right) P A^{m}=A^{m} P^{T}\left(\begin{array}{cccc}
K & K D & 0 & 0 \\
0 & 0 & 0 & 0 \\
0 & 0 & 0 & 0 \\
0 & 0 & 0 & 0
\end{array}\right) P
$$

COROllary 3. Under the hypothesis of Theorem 2, if $A$ is also range-Hermitian, that is, $A^{\#}=A^{\dagger}$, then $A^{\dagger}=A^{\#}=H A^{m}=A^{m} H$ where $H$ is a diagonal matrix with positive diagonal entries and $m$ is a positive integer.

Proor. Let $A$ be range-Hermitian. Then $A^{\dagger}=A^{\#}$. By Theorem 1,

$$
P A P^{T}=\left(\begin{array}{cccc}
J & J D & 0 & 0 \\
0 & 0 & 0 & 0 \\
C J & C J D & 0 & 0 \\
0 & 0 & 0 & 0
\end{array}\right)
$$

where $J$ is a direct sum of matrices of types (I) or (II). But then by using $A^{\dagger}=A^{\#}$, we obtain that $C$ and $D$ must be zero. Thus

$$
P A P^{T}=\left(\begin{array}{ll}
J & 0 \\
0 & 0
\end{array}\right)
$$


and

$$
\begin{aligned}
A^{\#} & =P^{T}\left(\begin{array}{cc}
K & 0 \\
0 & 0
\end{array}\right) P A^{m}=A^{m} P^{T}\left(\begin{array}{cc}
K & 0 \\
0 & 0
\end{array}\right) P \\
& =P^{T}\left(\begin{array}{ll}
K & 0 \\
0 & 0
\end{array}\right)\left(\begin{array}{cc}
J^{m} & 0 \\
0 & 0
\end{array}\right) P=P^{T}\left(\begin{array}{cc}
J^{m} & 0 \\
0 & 0
\end{array}\right)\left(\begin{array}{cc}
K & 0 \\
0 & 0
\end{array}\right) P \\
& =P^{T}\left(\begin{array}{ll}
K & 0 \\
0 & I
\end{array}\right)\left(\begin{array}{cc}
J^{m} & 0 \\
0 & 0
\end{array}\right) P=P^{T}\left(\begin{array}{cc}
J^{m} & 0 \\
0 & 0
\end{array}\right)\left(\begin{array}{cc}
K & 0 \\
0 & I
\end{array}\right) P \\
& =P^{T}\left(\begin{array}{ll}
K & 0 \\
0 & I
\end{array}\right) P A^{m}=A^{m} P^{T}\left(\begin{array}{cc}
K & 0 \\
0 & I
\end{array}\right) P \\
& =H A^{m}=A^{m} H,
\end{aligned}
$$

where $H$ is a diagonal matrix with positive diagonal entries.

Before we prove Corollary 4 we record below a simple fact which we state without proof.

SUBLEMMA. If $\beta x y^{T}$ is a row (or column) stochastic matrix where $\beta>0$ and $x, y$ are positive vectors such that $y^{T} x=1$ then $\beta=1$.

COROLlaRy 4. If $A$ is an $n \times n$ nonnegative row (or column) stochastic matrix such that $A^{(1,2)}=p(A)>0, p(A)$ is a polynomial in $A$ with scalar coefficients, then $A^{(1,2)}=A^{m}$ for some positive integer $m$ and $A^{(1,2)}$ is row (or column) stochastic.

Proof. For definiteness, let us assume that $A$ is row stochastic. By appropriate application of Lemma 1 and Theorem 1, we can find a permutation matrix $\boldsymbol{P}$ such that

$$
P A P^{T}=\left(\begin{array}{cc}
J & 0 \\
C J & 0
\end{array}\right)
$$

where $J$ is a direct sum of matrices of types (I) or (II) (not necessarily both). We note that if $S$ is a summand of type (I) then by sublemma $\beta=1$ and so $S^{(1,2)}=x y^{T}=S$. Next, let $S$ be a summand of type (II). Then $S$ is a stochastic matrix and

$$
S=\left(\begin{array}{cccccc}
0 & \beta_{12} x_{1} y_{2}^{T} & 0 & 0 & \ldots & 0 \\
0 & 0 & \beta_{23} x_{2} y_{3}^{T} & 0 & \ldots & 0 \\
\ldots & 0 & 0 & \ldots & 0 & \beta_{d-1 d} x_{d-1} y_{d}^{T} \\
\beta_{d 1} x_{d} y_{1}^{T} & 0 & 0 & \ldots & 0 & 0
\end{array}\right),
$$

where $\beta_{i j}>0$ and $x_{i}, y_{i}$ are positive vectors with $y_{i}{ }^{r} x_{i}=1$. A straightforward verification shows that $S^{(1,2)}=\left(\beta_{12} \beta_{23} \cdots \beta_{d 1}\right)^{-1} S^{d-1}$ and $S^{d-1}$ is a row stochastic matrix. Thus again by the sublemma we get $\left(\beta_{12} \beta_{23} \cdots \beta_{d 1}\right)=1$. Then as in the proof of Theorem 2, we get $J^{(1,2)}=J^{m}$, and hence

$$
A^{(1,2)}=P^{T}\left(\begin{array}{cc}
J^{m} & 0 \\
C J^{m} & 0
\end{array}\right) P=A^{m} .
$$


THEOREM 3. Every nonnegative rank factorization of nonnegative matrices $J$ which are direct sum of matrices of types $\left(\mathrm{I}^{\prime}\right)$ or $\left(\mathrm{II}^{\prime}\right)$ (not necessarily both) is of the form $J=(F Q)\left(Q^{T} G\right)$, where $Q$ is a permutation matrix, $F$ and $G$ are respectively the direct sum of matrices of the form (1) or (2) and (1') or (2'):

(1) $\beta^{\prime} x$,

(1') $\beta^{\prime \prime} y^{T}$,

(2)

$$
\left(\begin{array}{cccccc}
0 & \gamma_{1} x_{1} & 0 & 0 & \cdots & 0 \\
0 & 0 & \gamma_{2} x_{2} & 0 & \cdots & 0 \\
0 & 0 & 0 & \cdots & 0 & \gamma_{d-1} x_{d-1} \\
\gamma_{d} x_{d} & 0 & 0 & \cdots & 0 & 0
\end{array}\right)
$$

and

(2')

$$
\left(\begin{array}{cccc}
\gamma_{1}^{\prime} y_{1}^{T} & & & 0 \\
& \gamma_{2}^{\prime} y_{2}^{T} & & \\
& & \ddots & \\
0 & & & \gamma_{d}^{\prime} y_{d}^{T}
\end{array}\right)
$$

such that $\beta^{\prime}>0, \beta^{\prime \prime}>0, \gamma_{i}>0, \gamma_{i}^{\prime}>0, x, y, x_{i}, y_{i}$ are positive vectors with $y^{T} x=1$ and $y_{i}^{T} x_{i}=1$. Moreover, $J^{\#}=p(J)$ where $p(t)=\sum_{i=1}^{k} \alpha_{i} t^{m_{1}}, \alpha_{i} \neq 0, m_{i}>$ 0 , is some polynomial in $t, \beta^{\prime} \beta^{\prime \prime}$ is a root of equation (6), and the product $\gamma_{1} \gamma_{2} \cdots \gamma_{d} \gamma_{1}^{\prime} \gamma_{2}^{\prime} \cdots \gamma_{d}^{\prime}$ is a common root of the system of at most $d$ equations (7) and (8). It is understood that in forming the product $(F Q)\left(Q^{T} G\right)$ if $F$ has a summand of type (1) or (2) in the ith place of its direct sum then $G$ has a corresponding summand of type (1') or (2') at the same ith place.

Also, for each nonnegative rank factorization $J=F G$ of $J,(G F)^{-1}=p(G F)$.

Proof. Let $S=\beta x y^{T}$ with $y^{T} x=1$ be a summand of $J$ of type (I'). Clearly, the only possible nonnegative rank factorization of $S$ is $S=F G, F=\beta^{\prime} x, G=\beta^{\prime \prime} y^{T}$ with $\beta^{\prime} \beta^{\prime \prime}=\beta$. This gives $G F=\beta y^{T} x=\beta$. By equation (6), it then follows that $(G F)^{-1}=p(G F)$.

Next let

$$
S=\left(\begin{array}{cccccc}
0 & \beta_{12} x_{1} y_{2}^{T} & 0 & 0 & \ldots & 0 \\
0 & 0 & \beta_{23} x_{2} y_{3}^{T} & 0 & \ldots & 0 \\
\cdots & \ldots & \ldots & \ldots & \ldots & \ldots \\
\beta_{d 1} x_{d} y_{1}^{T} & 0 & 0 & \ldots & 0 & 0
\end{array}\right]
$$


be a summand of $J$ of type (II') of rank $d$. Let $S=F G$ be a nonnegative rank factorization of $S$. Partition $F=\left(F_{i j}\right)$ and $G=\left(G_{i j}\right)$ into matrix blocks such that for all $i, j, k, F_{i j} G_{j k}$ is defined and is of the same order as that of the $(i, k)$ th block entry in $S$. Since $F$ is of full column rank, no column of $F$ is zero. Also no row of $F$ can be zero. For otherwise, $S=F G$ shall have a zero row which is not true. Similarly, no row or column of $G$ is zero. Since no column of $F$ is zero, for each $j$ there exists an $i$ (depending on $j$ ) such that $F_{i j} \neq 0$. But then $G_{j k}=0$ for all $k \neq i+1$. Thus each row of the partitioned matrix $G$ has at most one (and hence exactly one) nonzero entry. Clearly, then each column of $G$ has also exactly one nonzero entry. The same reason yields that the partitioned matrix $F$ has exactly one nonzero entry in each row and in each column. This implies there exists a permutation $\sigma \in S_{d}$ such that $F_{i j}=0$, for all $j \neq \sigma(i), G_{\sigma(i) k}=0$, for all $k \neq i+1$ and $F_{i \sigma(i)} G_{\sigma(i), i+1}=\beta_{i, i+1} x_{i} y_{i+1}^{T}$. But then the only solutions for $F_{i \sigma(i)}$ and $G_{\sigma(i), i+1}$ are given by $F_{i o(i)}=\gamma_{i} x_{i}$ and $G_{\sigma(i), i+1}=\gamma_{i}^{\prime} y_{i+1}^{T}$ where $\gamma_{i} \gamma_{i}^{\prime}=\beta_{i, i+1}$. It is now clear that $J=(F Q)\left(Q^{T} G\right)$ where $F$ and $G$ are, respectively, the direct sum of matrices of the form (1) or (2) and $\left(1^{\prime}\right)$ or $\left(2^{\prime}\right)$ and $Q$ is some permutation matrix. For summand of $J$ of type $\left(I^{\prime}\right)$ we have already shown that $(G F)^{-1}=p(G F)$. We now show the same for summand of type $\left(\mathrm{II}^{\prime}\right)$. It is sufficient to prove the result for $S=F G$ where

$$
F=\left(\begin{array}{cccccc}
0 & \gamma_{1} x_{1} & 0 & 0 & \ldots & 0 \\
0 & 0 & \gamma_{2} x_{2} & 0 & \ldots & 0 \\
\cdots & \ldots & \ldots & \ldots & \ldots & \ldots \\
0 & 0 & 0 & \cdots & 0 & \gamma_{d-1} x_{d-1} \\
\gamma_{d} x_{d} & 0 & 0 & \cdots & 0 & 0
\end{array}\right)
$$

and

$$
G=\left(\begin{array}{cccc}
\gamma_{1}^{\prime} y_{1}^{T} & & & 0 \\
& \gamma_{2}^{\prime} y_{2}^{T} & & \\
& & \ddots & \\
0 & & & \gamma_{d}^{\prime} y_{d}^{T}
\end{array}\right)
$$

Then

$$
G F=\left(\begin{array}{cccccc}
0 & \gamma_{1} \gamma_{1}^{\prime} & 0 & 0 & \ldots & 0 \\
0 & 0 & \gamma_{2} \gamma_{2}^{\prime} & 0 & \ldots & 0 \\
\cdots & \ldots & \ldots & \ldots & \ldots & \ldots \\
0 & 0 & 0 & \ldots & 0 & \gamma_{d-1} \gamma_{d-1}^{\prime} \\
\gamma_{d} \gamma_{d}^{\prime} & 0 & 0 & \ldots & 0 & 0
\end{array}\right)
$$

and $(G F)^{d}=\left(\gamma_{1} \gamma_{2} \cdots \gamma_{d} \gamma_{1}^{\prime} \gamma_{2}^{\prime} \cdots \gamma_{d}^{\prime}\right) I$.

From equations (7) and (8), it follows that $\left(\gamma_{1} \gamma_{2} \cdots \gamma_{d} \gamma_{1}^{\prime} \gamma_{2}^{\prime} \cdots \gamma_{d}^{\prime}\right)^{1 / d}$ is a root of $\sum_{i=1}^{k} \alpha_{i} t^{m_{i}+1}=1$. Thus $\sum_{i=1}^{k} \alpha_{i}(G F)^{m_{i}+1}=I$, since $(G F)^{d}=$ $\left(\gamma_{1} \gamma_{2} \cdots \gamma_{d} \gamma_{1}^{\prime} \gamma_{2}^{\prime} \cdots \gamma_{d}^{\prime}\right) I$. Hence $(G F) p(G F)=I$, completing the proof.

The next theorem describes all nonnegative rank factorizations of a nonnegative matrix with a nonnegative group inverse. 
TheOREM 4. (a) Let $A>0$ and $P$ be a permutation matrix such that

$$
P A P^{T}=\left(\begin{array}{cccc}
J & J D & 0 & 0 \\
0 & 0 & 0 & 0 \\
C J & C J D & 0 & 0 \\
0 & 0 & 0 & 0
\end{array}\right)
$$

$J$ is a direct sum of matrices of types $\left(\mathrm{I}^{\prime}\right)$ or $\left(\mathrm{II}^{\prime}\right), C, D$ are some nonnegative matrices of suitable sizes (equivalently, $A>0$ and $A^{\sharp}=p(A)>0$ ). Then we have the following:

(a) If $J=F G$ is a nonnegative rank factorization of $J$ then it "lifts" to a nonnegative rank factorization

$$
A=P^{T}\left(\begin{array}{c}
F \\
0 \\
C F \\
0
\end{array}\right]\left(\begin{array}{llll}
G & G D & 0 & 0
\end{array}\right) P
$$

of $A$.

(a) If $A=F^{\prime} G^{\prime}$ is a nonnegative rank factorization of $A$ then it "contracts" to a nonnegative rank factorization $J=F_{11}^{\prime} G_{11}^{\prime}$ of $J$ where $F_{11}^{\prime}\left(G_{11}^{\prime}\right)$ consists of first $n$ rows (columns) of $P F^{\prime}\left(G^{\prime} P^{T}\right), n$ being the order of the matrix $J$.

$\left(a_{3}\right)$ If $\sigma$ denotes the operation of "lifting" defined in $\left(a_{1}\right)$, and $\eta$ denotes the

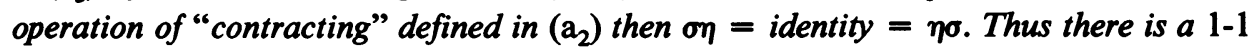
correspondence between the class of nonnegative rank factorizations of $J$ and the class of nonnegative rank factorizations of $A$.

$\left(\mathrm{a}_{4}\right)$ If $A=F^{\prime} G^{\prime}$ and $J=F G$ are corresponding nonnegative rank factorizations of $A$ and $J$, respectively, then $G F=G^{\prime} F^{\prime}$ and $(G F)^{-1}=p(G F)$, where $A^{\#}=p(A)$.

(b) If $A=F G$ is a nonnegative rank factorization of $A$ such that $(G F)^{-1}=p(G F)$ where $p(t)$ is some polynomial in $t$ with scalar coefficients then $A^{\#}$ exists, $A^{\sharp}>0$, and $A^{\#}=p(A)$.

Proof. $\left(a_{1}\right)$ Straightforward verification.

$\left(a_{2}\right)$ Let $n$ be the order of $J$. Partition

$$
P A P^{T}=\left(\begin{array}{cccc}
J & J D & 0 & 0 \\
0 & 0 & 0 & 0 \\
C J & C J D & 0 & 0 \\
0 & 0 & 0 & 0
\end{array}\right), \quad P F^{\prime}=\left(\begin{array}{c}
F_{11}^{\prime} \\
F_{21}^{\prime}
\end{array}\right), \quad \text { and } \quad G^{\prime} P^{T}=\left(G_{11}^{\prime} G_{12}^{\prime}\right)
$$

where $F_{11}^{\prime}\left(G_{11}^{\prime}\right)$ consists of first $n$ rows (columns) of $P F^{\prime}\left(G^{\prime} P^{T}\right)$ respectively. By comparing we get $J=F_{11}^{\prime} G_{11}^{\prime}$ which is clearly nonnegative rank factorization of $J$.

$\left(a_{3}\right)$ It is obvious that if we perform the operation of lifting followed by the operation of contracting then the composition is identity operation, i.e. $\eta \sigma=$ identity. On the other hand, it is not clear to us that o $\eta$ is also identity, in general. However, we can show o $\eta=$ identity under our hypothesis. Let $A=F^{\prime} G^{\prime}$ be a nonnegative rank factorization of $A$. By performing the operation $\eta$ we get 
$J=F_{11}^{\prime} G_{11}^{\prime}$ where $F_{11}^{\prime}$ and $G_{11}^{\prime}$ are respectively the first $n$ rows and the first $n$ columns of $P F^{\prime}$ and $G^{\prime} P^{T}$. Then by performing $\sigma$, we get

$$
A=P^{T}\left(\begin{array}{c}
F_{11}^{\prime} \\
0 \\
C F_{11}^{\prime} \\
0
\end{array}\right)\left(\begin{array}{llll}
G_{11}^{\prime} & G_{11}^{\prime} D & 0 & 0
\end{array}\right) P
$$

To prove $\sigma \eta=$ identity, we need to show

$$
P F^{\prime}=\left(\begin{array}{c}
F_{11}^{\prime} \\
0 \\
C F_{11}^{\prime} \\
0
\end{array}\right), \quad G^{\prime} P^{T}=\left(\begin{array}{llll}
G_{11}^{\prime} & G_{11}^{\prime} D & 0 & 0
\end{array}\right)
$$

Since by Theorem 3, $F_{11}^{\prime}\left(G_{11}^{\prime}\right)$ is the direct sum of matrices of the form (1) or (2) $\left(\left(1^{\prime}\right)\right.$ or $\left.\left(2^{\prime}\right)\right)$ it is sufficient to prove (9) when $F_{11}^{\prime}$ is of the form (1) or (2) and $G_{11}^{\prime}$ is of the corresponding form $\left(1^{\prime}\right)$ or $\left(2^{\prime}\right)$. Partition

$$
P F^{\prime}=\left(\begin{array}{l}
F_{11}^{\prime} \\
F_{21}^{\prime} \\
F_{31}^{\prime} \\
F_{41}^{\prime}
\end{array}\right), \quad G^{\prime} P^{T}=\left(\begin{array}{llll}
G_{11}^{\prime} & G_{12}^{\prime} & G_{13}^{\prime} & G_{14}^{\prime}
\end{array}\right)
$$

such that the size of $F_{j 1}^{\prime}, j=1,2,3,4$, is the same as that of the corresponding block in

$$
\left[\begin{array}{c}
F_{11}^{\prime} \\
0 \\
C F_{11}^{\prime} \\
0
\end{array}\right)
$$

Similarly, for the size of $G_{i j}^{\prime}, j=1,2,3,4$. Then comparing the corresponding blocks in

$$
\left[\begin{array}{c}
F_{11}^{\prime} \\
0 \\
C F_{11}^{\prime} \\
0
\end{array}\right)\left(\begin{array}{llll}
G_{11}^{\prime} & G_{11}^{\prime} D & 0 & 0
\end{array}\right)=\left(\begin{array}{c}
F_{11}^{\prime} \\
F_{21}^{\prime} \\
F_{31}^{\prime} \\
F_{41}^{\prime}
\end{array}\right)\left(\begin{array}{llll}
G_{11}^{\prime} & G_{12}^{\prime} & G_{13}^{\prime} & G_{14}^{\prime}
\end{array}\right)
$$

we get the result by observing that $F_{11}^{\prime}$ and $G_{11}^{\prime}$ are not zero divisors.

$\left(a_{4}\right)$ Follows from $\left(a_{1}\right)-\left(a_{3}\right)$ and Theorem 3.

(b) Recall that if $A=F G$ is a rank factorization of $A$ then $A^{\sharp}$ exists if and only if $(G F)^{-1}$ exists [4]. In this case $A^{\#}=F(G F)^{-2} G$. A straightforward computation then yields $A^{\#}=p(A)$ where $(G F)^{-1}=p(G F)$.

REMARK 2. Theorem 3 along with Theorem $4\left(a_{3}\right)$ characterizes all the nonnegative rank factorizations of a nonnegative matrix with nonnegative group inverse. 
REMARK 3. Another proof of Theorem $4\left(a_{4}\right)$ can be given on the same lines as the proof for the special case when $p(A)=A$ given by Berman and Plemmons [3]. However, the purpose of Theorem 4 is to characterize all nonnegative rank factorizations of nonnegative matrices $A$ with $A^{\sharp}>0$, and $\left(a_{4}\right)$ comes out as an offshoot.

\section{REFERENCES}

1. A. Ben-Israel and T. N. E. Greville, Generalized inverses: Theory and applications, Wiley, New York, 1974.

2. A. Berman, Nonnegative matrices which are equal to their generalized inverse, Linear Algebra and Appl. 9 (1974), 261-265.

3. A. Berman and R. J. Plemmons, Matrix group monotonicity, Proc. Amer. Math. Soc. 46 (1974), 355-359.

4. R. Cline, Inverses of rank invariant powers of a matrix, SIAM J. Numer. Anal. 5 (1968), 182-197. MR 37 \#2769.

5. R. DeMarr, Nonnegative idempotent matrices, Proc. Amer. Math. Soc. 45 (1974), 185-188.

6. P. Flor, On groups of nonnegative matrices, Compositio Math. 21 (1969), 376-382.

7. S. K. Jain, V. K. Goel and Edward K. Kwak, Nonnegative matrices having same nonnegative Moore-Penrase and group inverses, Linear and Multilinear Algebra 7 (1979), 59-72.

8. R. Penrose, A generalized inverse for matrices, Proc. Cambridge Philos. Soc. 51 (1955), 406-413.

Departmant of Mathematics, Ohio Universtry, Athens, Ohio 45701 (Current address of S. K. Jain)

Department of Mathematics and Computer Science, Wright State Univbrsity, Dayton, Ohio 45435

Current address (E. K. Kwak): Department of Mathematics, Central State University, Wilberforce, Ohio 45384

Current address (V. K. Goel): Simulation Technology, Inc., 4124 Linden Avenue, Dayton, Ohio 45432 